PHYSICAL REVIEW FLUIDS 1, 050510 (2016)

\title{
Shedding light on pilot-wave phenomena
}

\section{P.-T. Brun, Daniel M. Harris, Victor Prost, Julio Quintela, and John W. M. Bush* Department of Mathematics, Massachusetts Institute of Technology, \\ Cambridge, Massachusetts 02139, USA \\ (Received 12 August 2016; published 12 September 2016)}

This paper is associated with a video winner of a 2015 APS/DFD Gallery of Fluid Motion Award. The original video is available from the Gallery of Fluid Motion, http://dx.doi.org/10.1103/APS.DFD.2015.GFM.V0064

DOI: 10.1103/PhysRevFluids.1.050510

Droplets walking on a vibrating fluid bath, discovered over a decade ago [1], represent a macroscopic realization of the pilot-wave mechanics proposed for microscopic quantum particles

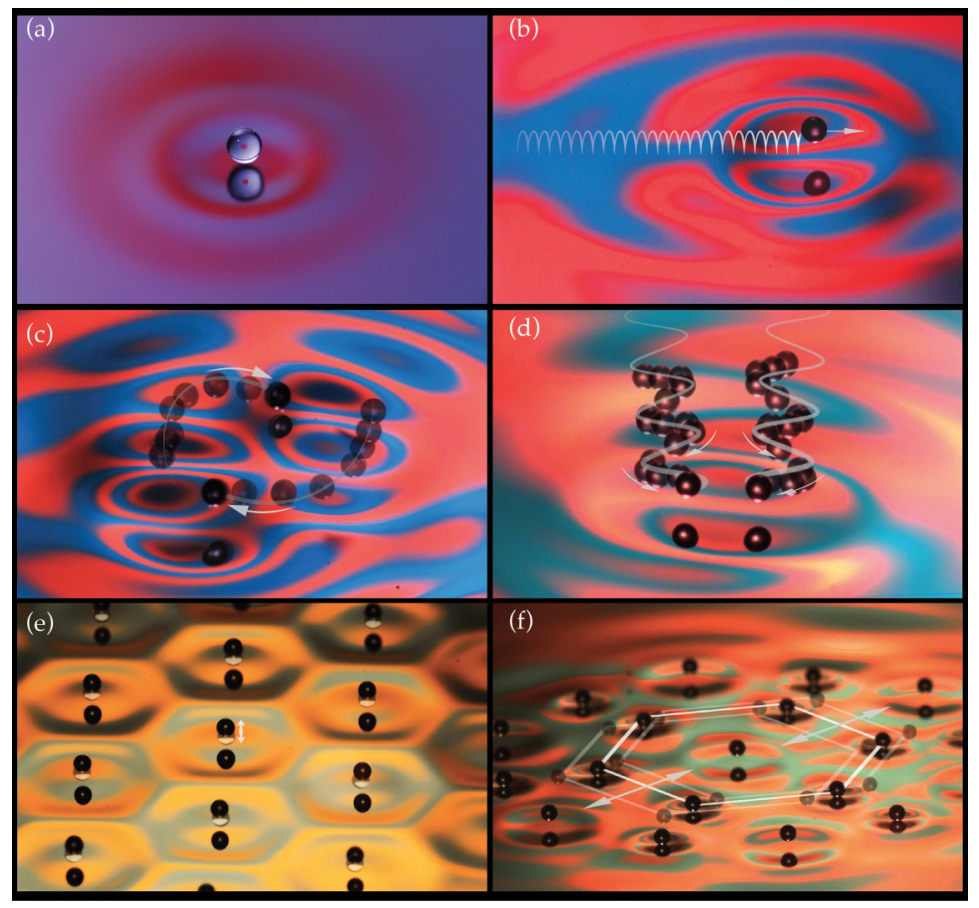

FIG. 1. (a) A droplet of an approximate diameter of $1 \mathrm{~mm}$ bounces on the surface of a vibrating oil bath. (b) For sufficiently large values of the vertical forcing, a droplet may spontaneously self-propel across the interface. Droplet pairs may interact through their wave fields, either (c) locking into orbits or (d) walking side by side in a promenade mode. (e) Multiple drops may organize into hexagonal lattices that (f) destabilize for sufficiently large values of the forcing acceleration. White streaks indicate the (b)-(e) complete or (c), (d), and (f) strobed droplet trajectories. DOI: http://dx.doi.org/10.1103/APS.DFD.2015.GFM.V0064

\footnotetext{
*Author to whom correspondence should be addressed: bush@math.mit.edu
}

Published by the American Physical Society under the terms of the Creative Commons Attribution 3.0 License. Further distribution of this work must maintain attribution to the author(s) and the published article's title, journal citation, and DOI. 


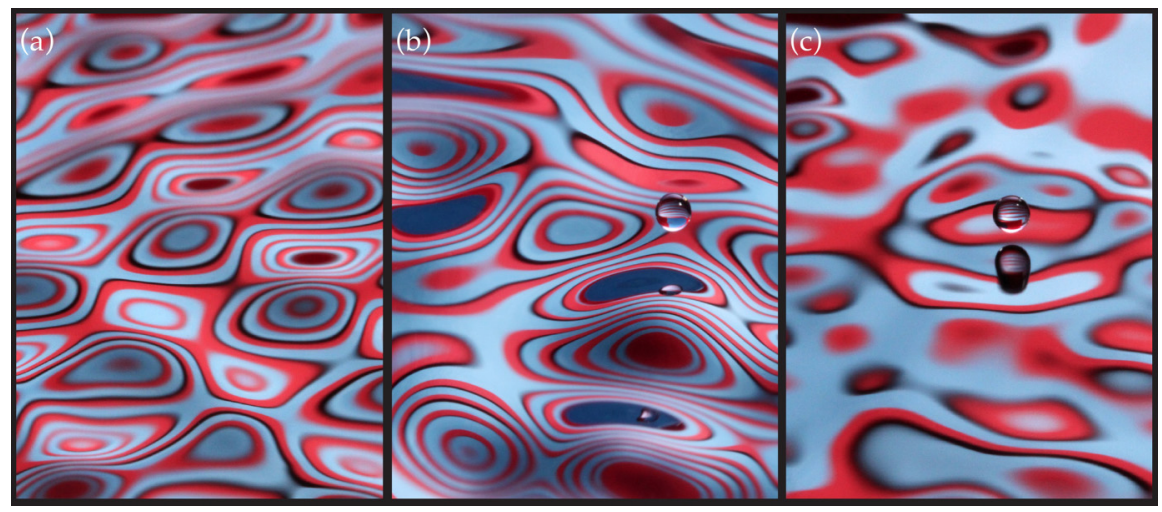

FIG. 2. (a) Faraday waves arise above a critical threshold in vibrational acceleration. (b) and (c) A droplet bouncing on the Faraday wave field moves in an unpredictable fashion and is at risk of coalescing. DOI: http://dx.doi.org/10.1103/APS.DFD.2015.GFM.V0064

by de Broglie [2]. This hydrodynamic pilot-wave system is of growing interest as it exhibits many of the peculiarities of quantum systems (see [3,4] for reviews).

We present here images of droplets bouncing on a vibrating bath. While the droplets are millimetric, the waves they generate have a characteristic amplitude of $10 \mu \mathrm{m}$ and so are not easily discernible with the naked eye. We use the experimental setup reported in Ref. [5] that includes a bath of 20-cSt oil vibrated sinusoidally at $80 \mathrm{~Hz}$. In order to illuminate the bath, a high-intensity light is placed behind striped colored patterns printed on paper and mounted on a light diffuser. The resulting images of the droplet and its pilot-wave field are captured with a high-speed camera.

Figure 1 has typical views of the bouncing droplets and accompanying wave fields when the bath vibration is below the Faraday threshold. Static bouncing and rectilinear walking states of single droplets are shown, as are bound states consisting of orbiting [6] or promenading [7] droplet pairs and stable and unstable crystal structures [8]. Above the Faraday threshold (Fig. 2), Faraday waves arise even in the absence of the droplet, so the droplet moves in an irregular fashion.

The authors gratefully acknowledge financial support from the NSF through Grant No. CMMI1333242 and the MIT-France Program. We also thank Sami Belkadi for contributing to Fig. 1(a), Juncal Arbelaiz and Emmanuel Siefert for contributing to Fig. 1(d) and Henry Kavle for contributing to Fig. 1(f).

[1] Y. Couder, S. Protiere, E. Fort, and A. Boudaoud, Dynamical phenomena: Walking and orbiting droplets, Nature (London) 437, 208 (2005).

[2] D. M. Harris and J. W. M. Bush, The pilot-wave dynamics of walking droplets, Phys. Fluids 25, 091112 (2013).

[3] J. W. M. Bush, Pilot-wave hydrodynamics, Annu. Rev. Fluid Mech. 47, 269 (2015).

[4] J. W. M. Bush, The new wave of pilot-wave theory, Phys. Today 68(8), 47 (2015).

[5] D. M. Harris, J. Quintela, V. Prost, P.-T. Brun, and J. W. M. Bush, Visualization of hydrodynamic pilot-wave phenomena, J. Visual. (2016), doi: 10.1007/s12650-016-0383-5 


\section{SHEDDING LIGHT ON PILOT-WAVE PHENOMENA}

[6] S. Protiere, S. Bohn, and Y. Couder, Exotic orbits of two interacting wave sources, Phys. Rev. E 78, 036204 (2008).

[7] C. Borghesi, J. Moukhtar, M. Labousse, A. Eddi, E. Fort, and Y. Couder, Interaction of two walkers: Wave-mediated energy and force, Phys. Rev. E 90, 063017 (2014).

[8] A. Eddi, A. Decelle, E. Fort, and Y. Couder, Archimedean lattices in the bound states of wave interacting particles, Europhys. Lett. 87, 56002 (2009). 